\title{
IMF dependence of Saturn's auroras: modelling study of HST and Cassini data from 12-15 February 2008
}

\author{
E. S. Belenkaya ${ }^{1}$, I. I. Alexeev ${ }^{1}$, M. S. Blokhina ${ }^{1}$, E. J. Bunce ${ }^{2}$, S. W. H. Cowley ${ }^{2}$, J. D. Nichols ${ }^{2}$, V. V. Kalegaev ${ }^{1}$, \\ V. G. Petrov ${ }^{3}$, and G. Provan ${ }^{2}$ \\ ${ }^{1}$ Institute of Nuclear Physics, Moscow State University, Vorob’evy Gory, 119992 Moscow, Russia \\ ${ }^{2}$ Department of Physics \& Astronomy, University of Leicester, Leicester LE1 7RH, UK \\ ${ }^{3}$ Pushkov Institute of Terrestrial Magnetism, Ionosphere and Radio Wave Propagation (IZMIRAN), Troitsk, Moscow Region, \\ 142190, Russia
}

Received: 10 February 2010 - Revised: 24 June 2010 - Accepted: 13 August 2010 - Published: 19 August 2010

\begin{abstract}
To gain better understanding of auroral processes in Saturn's magnetosphere, we compare ultraviolet (UV) auroral images obtained by the Hubble Space Telescope (HST) with the position of the open-closed field line boundary in the ionosphere calculated using a magnetic field model that employs Cassini measurements of the interplanetary magnetic field (IMF) as input. Following earlier related studies of pre-orbit insertion data from January 2004 when Cassini was located $\sim 1300$ Saturn radii away from the planet, here we investigate the interval 12-15 February 2008, when UV images of Saturn's southern dayside aurora were obtained by the HST while the Cassini spacecraft measured the IMF in the solar wind just upstream of the dayside bow shock. This configuration thus provides an opportunity, unique to date, to determine the IMF impinging on Saturn's magnetosphere during imaging observations, without the need to take account of extended and uncertain interplanetary propagation delays. The paraboloid model of Saturn's magnetosphere is then employed to calculate the magnetospheric magnetic field structure and ionospheric open-closed field line boundary for averaged IMF vectors that correspond, with appropriate response delays, to four HST images. We show that the IMF-dependent open field region calculated from the model agrees reasonably well with the area lying poleward of the UV emissions, thus supporting the view that the poleward boundary of Saturn's auroral oval in the dayside ionosphere lies adjacent to the open-closed field line boundary.
\end{abstract}

Keywords. Magnetospheric physics (Auroral phenomena; Planetary magnetospheres; Solar wind-magnetosphere interactions)

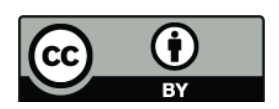

Correspondence to: E. S. Belenkaya (elena@dec1.sinp.msu.ru)

\section{Introduction}

Observations of Saturn's southern ultraviolet (UV) auroras using the Hubble Space Telescope (HST) have shown that they generally form a ring around each pole $\sim 1^{\circ}-3^{\circ}$ wide, located between $\sim 10^{\circ}-20^{\circ}$ co-latitude (e.g. Cowley et al., 2004a; Gérard et al., 2004; Grodent et al., 2005; Badman et al., 2006). While the dynamics of Saturn's magnetosphere are generally believed to be dominated by flows driven by the planet's rotation (e.g. Badman and Cowley, 2007), the auroras and related radio emissions are also known to respond strongly to increases in solar wind dynamic pressure that occur at interplanetary shocks (Clarke et al., 2005, 2009; Crary et al., 2005; Kurth et al., 2005; Jackman et al., 2005; Bunce et al., 2006; Badman et al., 2008). Saturn's system thus appears to combine aspects that are related both to the Earth, where auroral processes are driven primarily by solar wind-magnetosphere coupling (e.g. Milan et al., 2008), and to Jupiter, where they are related primarily to corotation enforcement currents flowing in the middle magnetosphere (e.g. Cowley and Bunce, 2001; Nichols and Cowley, 2004). Cowley and Bunce (2003) showed theoretically, however, that the latter currents at Saturn are too weak to produce bright auroras, and occur at latitudes that are too low to explain the observed emissions. Instead, Cowley et al. (2004a, b) suggested that the oval is associated with corotation breakdown in the vicinity of the open-closed field line boundary, such that the auroras would also be subject to solar wind modulation, for example through changes in the amount of open flux present in the system (Cowley et al., 2005; Badman et al., 2005). Subsequently, Bunce et al. (2008) employed simultaneous HST images and high-latitude data from the Cassini spacecraft to show that the aurora near noon is indeed located near the boundary between open and closed field lines, where a layer of upward-directed field-aligned

Published by Copernicus Publications on behalf of the European Geosciences Union. 
current flows whose density requires downward acceleration of magnetospheric electrons sufficient to produce the aurora. Further theoretical modelling of the current system related to these results has been presented by Cowley et al. (2008).

HST observations of Saturn's auroras in January 2004, combined with simultaneous observations of the interplanetary medium by Cassini then inbound to the planet prior to orbit insertion (July 2004), showed that the auroral emissions brighten at the time of large shock-related solar wind pressure and interplanetary magnetic field (IMF) magnitude increases (Clarke et al., 2005; Crary et al., 2005; Cowley et al., 2005; Bunce et al., 2006). Further events of this nature have been studied in data obtained in subsequent HST campaigns in January-February 2007 and in February 2008 (Clarke et al., 2009). Such auroral brightenings occur most often in the dawn sector, where the poleward boundary of the bright emissions contracts such that the auroras fill much of the polar cap on the dawn side. These auroral brightenings can last for hours to days, in some cases longer than the duration of the solar wind pressure increase that caused it. Belenkaya et al. $(2007,2008)$ used magnetospheric magnetic field models incorporating lagged Cassini magnetic field measurements to study the January 2004 events, the results indicating that changes in the IMF also lead to variations in the size and position of the open field region in the southern polar ionosphere that are reflected in the aurora. However, determination of the detailed correspondence between auroral images and related interplanetary conditions using this data set presents challenges, due to the considerable distance between the spacecraft and the planet during the interval. Specifically, during this campaign Cassini was located near the ecliptic plane $\sim 500 R_{\mathrm{S}}$ upstream from the planet and $\sim 1200 R_{\mathrm{S}}$ towards dawn $\left(R_{\mathrm{S}}\right.$ is Saturn's equatorial radius, equal to $60268 \mathrm{~km}$ ). The latter dawnward displacement in particular yields propagation uncertainties of several hours in the nominal radial propagation delay of $\sim 17 \mathrm{~h}$, due to possible non-radial normal alignment and propagation of the solar wind disturbances.

In this paper we further investigate the relationship between Saturn's aurora and the modelled open-closed field boundary, but now using a subsequent data set in which the inherent timing uncertainties are much reduced. Specifically, we study an interval, unique to date, in which HST imaging was coordinated with simultaneous interplanetary observations by Cassini located immediately upstream of Saturn's bow shock. The interval is $12-15$ February 2008, corresponding to day of year (DOY) 43-46 inclusive. The Cassini magnetic field observations are used to provide suitably lagged IMF data which are employed within the Saturn paraboloid magnetosphere model (Alexeev et al., 2006; Belenkaya et al., 2006a, b, 2007, 2008), to calculate the position of the open-closed field line boundary in Saturn's ionosphere. The calculated boundary is then compared with the Southern Hemisphere UV auroral images obtained by the HST.

\section{Data for DOY 43-46 2008}

UV images of Saturn's southern aurora were obtained by the HST in February 2008 on an approximately daily basis from DOY 32 to 47 , as discussed in detail by Clarke et al. (2009). For much of this interval Cassini was located inside Saturn's magnetosphere, but it emerged into the solar wind near apoapsis on the dayside of the planet during DOY 43-46, which is thus the interval studied here. Solar wind parameters propagated from near-Earth measurements indicate that an interplanetary shock arrived at Saturn in the middle of day 43 across which the solar wind speed increased from 440 to $520 \mathrm{~km} \mathrm{~s}^{-1}$ (Clarke et al., 2009). Following this, the solar wind speed increased further to near $600 \mathrm{~km} \mathrm{~s}^{-1}$ on DOY 45, declining slowly on DOY 46. Correspondingly, the propagated solar wind dynamic pressure increased from values of $\sim 0.02 \mathrm{nPa}$ following the shock to peak near $\sim 0.1 \mathrm{nPa}$ on days 44 and 45 , before declining to smaller values again towards the end of the interval.

Figure 1 shows the Cassini trajectory in kronocentric solar magnetospheric (KSM) coordinates from near the periapsis of revolution (Rev) 58 on DOY 39 to near the periapsis of Rev 59 on DOY 51, thus containing the interval DOY 43-46 of interest here. In the KSM system X points towards the Sun (approximately anti-parallel to the solar wind flow), the $\mathrm{X}-\mathrm{Z}$ plane contains the planet's magnetic (and spin) axis, and $\mathrm{Y}$ completes the right-hand orthogonal triad pointing towards dusk. From top to bottom, the three panels of the figure show the trajectory projected onto the $\mathrm{X}-\mathrm{Y}, \mathrm{X}-\mathrm{Z}$, and $\mathrm{Y}-\mathrm{Z}$ planes, with the position marked by stars at the beginning of each even-numbered day. The solid and dashed red lines show the intersections of the magnetopause and bow shock, respectively, with these planes, derived from the empirical models of Kanani et al. (2010) and Masters et al. (2008), respectively. The models are shown for a solar wind dynamic pressure of $0.1 \mathrm{nPa}$ characteristic of peak expected values during the interval of interest, thus showing that the spacecraft is expected to have emerged from the magnetosphere and magnetosheath into the solar wind during the apoapsis interval.

That this is indeed the case is shown in Fig. 2, where we plot magnetic field data obtained by the Cassini fluxgate magnetometer (Dougherty et al., 2004) for DOY 43-46, corresponding to the blue trajectory segment spanning apoapsis in Fig. 1. Specifically, we show the three components of the magnetic field in KSM coordinates and the field magnitude, together with a colour-coded region identifier at the top of the plot. In the latter panel green corresponds to the magnetosphere, red to the magnetosheath, and blue to the solar wind. Position information is given at the bottom of the figure, namely the spacecraft radial distance $\left(R_{\mathrm{S}}\right)$, latitude (deg), and local time (h). Principal bow shock crossings (redblue transitions) are identified by vertical black dashed lines. It can be seen that the spacecraft was consistently located in the solar wind just upstream of Saturn's dayside bow shock from the middle of DOY 43 to the end of DOY 45, except for 

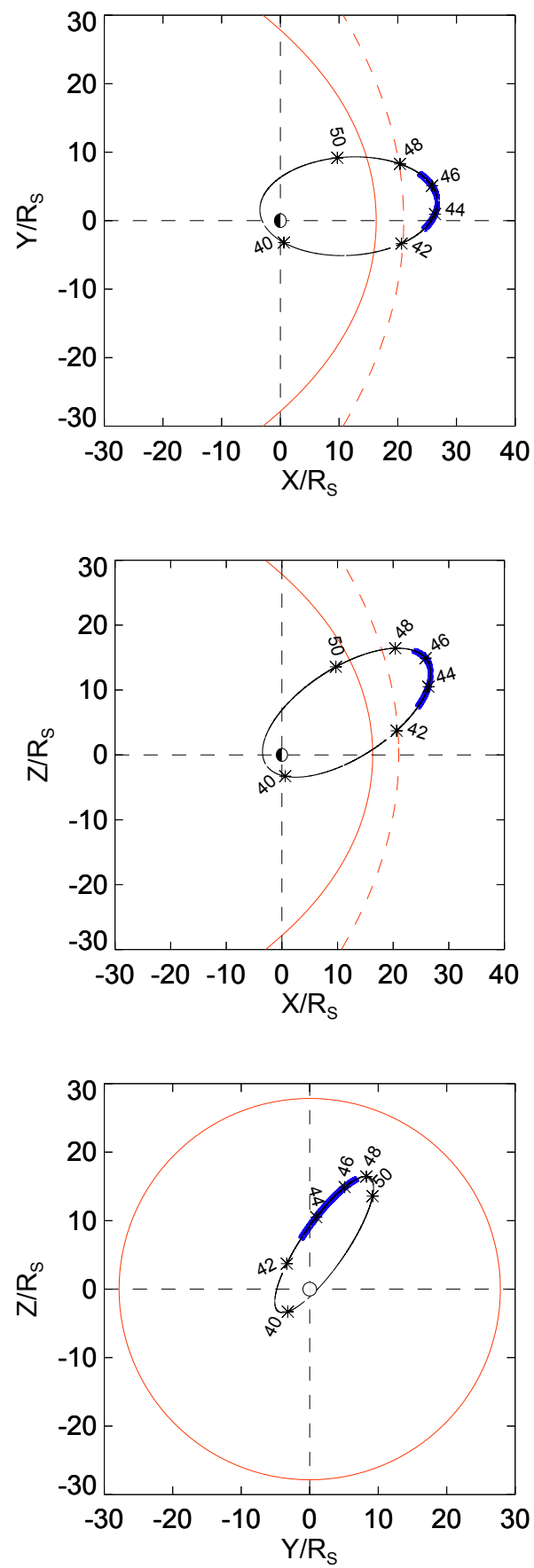

Fig. 1. Trajectory of the Cassini spacecraft in KSM coordinates from near the periapsis of Rev 58 to near the periapsis of Rev 59 . The three panels show from top to bottom the trajectory projected onto the $\mathrm{X}-\mathrm{Y}, \mathrm{X}-\mathrm{Z}$, and $\mathrm{Y}-\mathrm{Z}$ planes, with the position marked by stars at the beginning of each even-numbered day. The intersections of the magnetopause and bow shock with these planes are shown by the solid and dashed red lines, respectively, obtained from the models of Kanani et al. (2010) and Masters et al. (2008) for a solar wind dynamic pressure of $0.1 \mathrm{nPa}$. The blue segment of the trajectory spanning apoapsis corresponds to the interval for which magnetic field data are shown in Fig. 2. a short interval in the first half of DOY 44 as marked. The IMF strength in the solar wind declined from $\sim 1 \mathrm{nT}$ near the beginning of the interval to $\sim 0.25 \mathrm{nT}$ towards its end. The blue vertical stripes labelled "A" to " $\mathrm{D}$ " correspond to the times of four HST imaging intervals to be investigated below, which have been suitably lagged in time, as we now go on to discuss.

In order to study the IMF influence on Saturn's aurora by comparison of magnetic model results with HST images, we need first to determine the appropriate interval in the solar wind that corresponds to a particular image, taking account of propagation and response time effects. Three such time delays must be considered. The first, $\tau_{1 \mathrm{~S}}$, is the one-way light travel time between Saturn at the time of emission and the HST position at the time the image was obtained. For the period studied this time delay is about $1 \mathrm{~h} 10 \mathrm{~min}$. The second time delay, $\tau_{2 S}$, is the solar wind propagation time between Cassini and the reconnection regions on Saturn's magnetopause, corresponding to the subsolar region for northward IMF, and the cusp region for southward IMF (noting that these are opposite to the case of the Earth due to the opposite polarity of Saturn's magnetic field). For the period studied the distance between Cassini and these reconnection sites was approximately 10-20 $R_{\mathrm{S}}$, such that for a typical solar wind speed of $\sim 500 \mathrm{~km} \mathrm{~s}^{-1}$ the value of $\tau_{2 \mathrm{~S}}$ should be $\sim 30$ min (compared with around $17 \mathrm{~h}$ for the January 2004 campaign data). The third time delay, $\tau_{3 \mathrm{~S}}$, which is more difficult to estimate, is the auroral and flow response time in Saturn's ionosphere resulting from changes in the IMF following their arrival at the magnetopause. This includes the time scale to re-configure the near-planet magnetosphere leading to the establishment of a new polar convection pattern, and the time to communicate those changes along the field to the ionosphere. If we assume these processes are similar to those at the Earth, and propagate with similar velocities related to the solar wind speed and the Alfvén speed in the outer magnetosphere (the latter being similar in the two systems to within a modest factor), the response times in the two cases should then scale approximately with the spatial size of the systems. We should then multiply the corresponding time delay for the Earth, $\tau_{3 \mathrm{E}}$, by the factor $\left(R_{\mathrm{S}} / R_{\mathrm{E}}\right)\left(R_{\mathrm{SS}} / R_{\mathrm{SE}}\right) \approx 16.5$, where $R_{\mathrm{E}}$ is the Earth's radius $(6378 \mathrm{~km})$, and $R_{\mathrm{SS}}$ and $R_{\mathrm{SE}}$ are the subsolar magnetopause distances for Saturn and Earth in planetary radii, respectively ( $R_{\mathrm{SE}} \sim 10 R_{\mathrm{E}}$ and $R_{\mathrm{SS}} \sim 17.5 R_{\mathrm{S}}$ for the case studied, as will be shown below). In other words, we assume that $\tau_{3 \mathrm{E}}$ scales with the size of the magnetosphere. However, for the case of the Earth a broad range of $\tau_{3 \mathrm{E}}$ estimations have been obtained from various studies, as we now review.

Rishbeth et al. (1985) estimated the time delay for the Earth's ionospheric flow response to changes in the IMF using simultaneous upstream field observations by the AMPTE-UKS satellite and ionospheric plasma flow measurements by the EISCAT radar. They discussed one event in which a sharp southward turning of the IMF was followed 


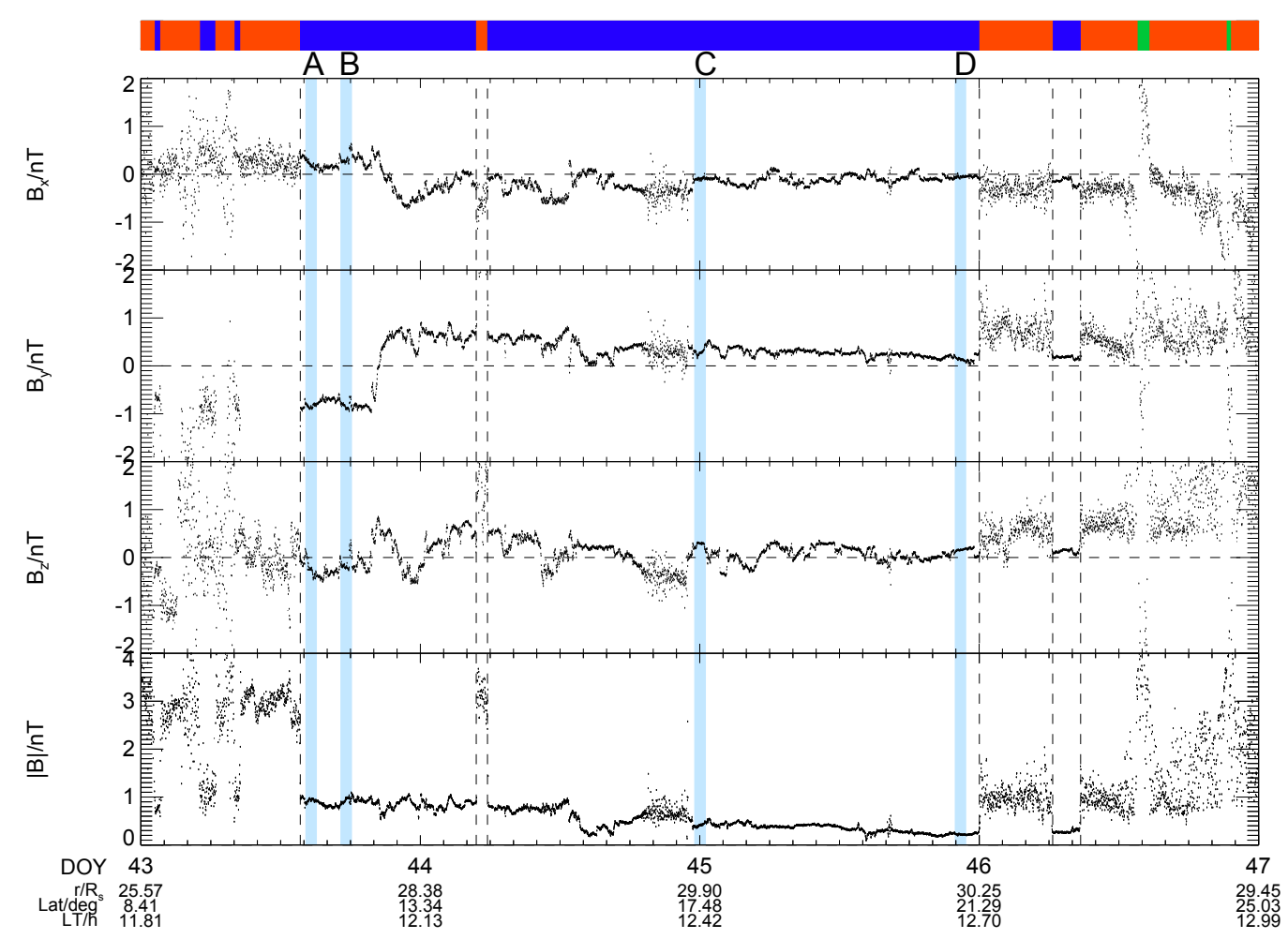

Fig. 2. Magnetic field data for DOY 43 to 46 of 2008, corresponding to the blue trajectory segment spanning apoapsis in Fig. 1. From top to bottom the data panels show the three components of the magnetic field in KSM coordinates, and the field magnitude. In the colour-coded region identifier at the top of the plot green corresponds to the magnetosphere, red to the magnetosheath, and blue to the solar wind. Principal bow shock crossings (red-blue transitions) are identified by vertical black dashed lines. The data at the bottom of the plot give the radial distance $\left(R_{\mathrm{S}}\right)$, latitude $(\mathrm{deg})$, and local time $(\mathrm{h})$ of the spacecraft. The blue vertical stripes labelled "A" to "D" correspond to the suitably lagged times of four HST imaging intervals.

by the onset of rapid ionospheric plasma flow near local noon with a delay of $13 \mathrm{~min}$. Using similar data, Todd et al. (1988) found a rapid response of the high-latitude ionospheric flow near noon on time scales no longer than a few minutes, with the effect subsequently propagating to other local times on time scales of about $10 \mathrm{~min}$. The ionospheric response delay following northward turnings was found to be similar to that following southward turnings. Hairston and Heelis (1995), using solar wind data from the IMP-8 spacecraft and ionospheric convection data from the DMSP satellites, measured response times of 17 to $25 \mathrm{~min}$ for north-to-south turnings of the IMF and longer lags of between 28 to $44 \mathrm{~min}$ for south-tonorth turnings. The difference in the time scales in the studies of Todd et al. (1988) and Hairston and Heelis (1995) could be attributed to the method of observation, whether focusing on the local initial response or the longer time required for the changed flow pattern to become fully established. For example, Clauer and Friis-Christensen (1988) observed the initial convection change to occur about 3 min following an IMF northward turning, while the final convection reconfiguration took an additional $20 \mathrm{~min}$. Taylor et al. (1994) studied the response of the high-latitude ionospheric convection flow measured by radars to southward turnings of the IMF, and found a response time on the dayside of $\sim 15-30 \mathrm{~min}$. Ridley et al. (1997) using the ionospheric electrodynamics (AMIE) technique computed the ionospheric electric potential over intervals of non-steady flow. Investigating its dynamics they determined the amount of time the ionosphere takes to reconfigure after a change in IMF orientation, finding that the penetrating electric field is applied across the entire polar cap boundary on a very rapid timescale (a few seconds). They concluded that the time delay for overall ionospheric reconfiguration is between 12 and $24 \mathrm{~min}$, if the IMF change takes $50 \mathrm{~min}$ or less. Huang et al. (2000) have also analyzed ionospheric convection patterns and their response to IMF changes. They found that the ionospheric flows begin to change 5-9 $( \pm 3)$ min later than the estimated arrival of the IMF $B_{Z}$ changes at the subsolar magnetopause. The ionospheric convection then takes an additional 10-20 min to reconfigure. Even from this list of results, which is far from complete, we see that there is no unique estimate of $\tau_{3 \mathrm{E}}$, such that the uncertainty in its value is rather large. Since Saturn is much less studied than the Earth, it is obvious that the uncertainty in this time is even larger. 
From this short review, however, it can be seen that two characteristic polar ionosphere response time scales to IMF changes can be identified. The initial response of the dayside flow and aurora occur on short time scales following transport in the solar wind plasma and Alfvén wave propagation down to the ionosphere, the latter being just a few minutes in the terrestrial system. The overall reconfiguration response time is a significantly longer interval, however, that we estimate for Earth to be $\tau_{3 \mathrm{E}} \sim 20 \mathrm{~min}$, of which the Alfvén communication time represents $\sim 10-20 \%$ of the total. From the above discussion we thus estimate for Saturn that $\tau_{3 \mathrm{~S}} \sim 16.5 \tau_{3 \mathrm{E}} \sim 330 \mathrm{~min}$, such that the overall delay from solar wind observation to ionosphere is $\tau_{2 S}+\tau_{3 S} \sim 6 \mathrm{~h}$. This delay, together with the light propagation time to Earth, is thus adopted here to find the Cassini solar wind interval corresponding to a particular HST image. We have then averaged the IMF data over a $1 \mathrm{~h}$ interval centred on this time in order to obtain a reasonable representative value of the IMF relating to each image.

\section{Paraboloid model calculations for 12-15 February 2008}

As indicated above, the location of the open-closed field line boundary in the ionosphere for given interplanetary conditions is determined here via field line calculations using the Saturn magnetospheric model described by Alexeev et al. (2006) and Belenkaya et al. (2006b, 2007, 2008), in which the magnetopause is taken to be a paraboloid of revolution about the Saturn-Sun line. The model is consequently expressed in KSM coordinates. The main contributors to the model magnetic field are (i) the intrinsic magnetic (dipole) field of the planet, together with the shielding magnetopause current which confines the dipole field inside the magnetopause, (ii) the tail currents and their closure currents on the magnetopause, (iii) the ring current and the corresponding shielding magnetopause current, and (iv) the IMF which penetrates into the magnetosphere.

The parameters which define Saturn's magnetospheric magnetic field in the model are then as follows (see above references for details): (i) $R_{\mathrm{SS}}$ is the distance from Saturn's centre to the subsolar point on the magnetopause; (ii) $R_{\mathrm{rc} 1}$ and $R_{\mathrm{rc} 2}$ are the distances to the outer and inner edges of the ring current, respectively; (iii) $R_{2}$ is the distance from the planet's centre to the inner edge of the magnetospheric tail current sheet; (iv) the field magnitude of the tail currents at the inner edge of the tail current sheet is $B_{\mathrm{t}} / \alpha_{0}$, where $\alpha_{0}=\left(1+2 R_{2} / R_{\mathrm{SS}}\right)^{1 / 2}$; (v) $\Psi$ is the tilt angle between the magnetic dipole axis and the KSM Z axis (approximately $-8.4^{\circ}$ during DOY 43-46 2008); and (vi) $B_{\mathrm{rc} 1}$ is the radial component of the ring current magnetic field at the outer edge of the ring current. The effect of the IMF inside the magnetosphere is given by adding the uniform field $k_{\mathrm{S}} \boldsymbol{B}_{\mathrm{IMF}}$, where $\boldsymbol{B}_{\mathrm{IMF}}$ is the IMF vector and $k_{\mathrm{S}}$ is the coefficient of its penetration into the magnetosphere. The latter coefficient is not well determined for Saturn, but here we consider values of 0.2 and 0.8 which span the likely range, as discussed previously by Belenkaya et al. (2007).

In performing these calculations it is desirable to keep the basic model parameters fixed so that we can clearly discern the effect on the open field region of the variable direction of the IMF. Since according to the results presented by Clarke et al. (2009) the interval studied corresponds overall to one in which Saturn's magnetosphere was subject to compression by the solar wind, with the dynamic pressure peaking at $\sim 0.1 \mathrm{nPa}$ on DOY 44 and 45 , here we choose to use a relatively compressed model throughout. Specifically we employ the model derived originally by Belenkaya et al. (2006b) to describe the field during the Pioneer11 flyby, corresponding to a dynamic pressure of $\sim 0.08 \mathrm{nPa}$, which was subsequently successfully employed by Belenkaya et al. (2007) to describe Saturn's magnetosphere under high solar wind dynamic pressure conditions. The corresponding set of model parameters is $R_{\mathrm{SS}}=17.5 R_{\mathrm{S}}, R_{\mathrm{rc} 1}=12.5 R_{\mathrm{S}}$, $R_{\mathrm{rc} 2}=6.5 R_{\mathrm{S}}, B_{\mathrm{rc} 1}=3.62 \mathrm{nT}, R_{2}=14 R_{\mathrm{S}}$, and $B_{\mathrm{t}}=8.7 \mathrm{nT}$. We note, however, that the propagated solar wind dynamic pressures presented by Clarke et al. (2009) indicate that somewhat more moderate values, $\sim 0.02-0.04 \mathrm{nPa}$, are appropriate towards the beginning and end of the interval considered. To test the sensitivity of our results to the magnetosphere model employed, we have therefore repeated the calculations using a model derived by Belenkaya et al. (2008) which is appropriate to an intermediate pressure of $0.03 \mathrm{nPa}$, corresponding to more typical values of the magnetopause subsolar radius of $\sim 22 R_{\mathrm{S}}$. The modified calculations show that the results are not sensitively dependent on which model is employed, such that only the compressed model results will be shown here.

We initially investigate the influence of each KSM component of the IMF on the open field line region in the Southern Hemisphere, with the results shown in Fig. 3. In these plots noon is on the right and dusk at the top, and for definiteness we have taken the IMF penetration coefficient $k_{\mathrm{S}}=0.8$ in all cases. From top to bottom these plots show the influence of $B_{\mathrm{x}}$ for positive and negative $B_{\mathrm{Z}}$, and effect of $B_{\mathrm{y}}$ for positive and negative $B_{\mathrm{Z}}$. We see that $B_{\mathrm{X}}<0\left(B_{\mathrm{X}}>0\right)$ shifts the open field line region in the Southern Hemisphere towards noon (midnight), and that this effect is more pronounced for $B_{\mathrm{Z}}<0$. It can also be seen that $B_{\mathrm{Z}}<0$ significantly decreases the size of the open field region compared with $B_{\mathrm{Z}}>0$, especially for $B_{\mathrm{x}}>0$. The IMF $B_{\mathrm{y}}$ field then produces dawn-dusk asymmetries in the open field region, the latter being shifted to dusk (dawn) for $B_{\mathrm{y}}<0\left(B_{\mathrm{y}}>0\right)$. Again, the shift is most noticeable for $B_{\mathrm{Z}}<0$.

We now consider more specifically the results that apply for the IMF vectors appropriate to each HST image. Four such images are available, labelled "A" to " $D$ ", each obtained by the HST over an interval of $\sim 20$ min on DOY $43-46$ as described in more detail below. The start and end times of 
Table 1. For each HST image we give from left to right the start and the end times of the HST imaging interval (DOY/UT h:min), the start and end times of the light emission at Saturn, the lagged IMF times as discussed in the text, and the corresponding $1 \mathrm{~h}$ averaged KSM IMF components (nT).

\begin{tabular}{|c|c|c|c|c|c|c|c|c|c|}
\hline Image & $\begin{array}{l}\text { HST start DOY } \\
\text { UT h:min }\end{array}$ & $\begin{array}{l}\text { HST end DOY } \\
\text { UT h:min }\end{array}$ & $\begin{array}{c}\text { Saturn start DOY } \\
\text { UT h:min }\end{array}$ & $\begin{array}{c}\text { Saturn end DOY } \\
\text { UT h:min }\end{array}$ & $\begin{array}{l}\text { IMF start DOY } \\
\text { UT h:min }\end{array}$ & $\begin{array}{l}\text { IMF end DOY } \\
\text { UT h:min }\end{array}$ & $\begin{array}{r}<B_{\mathrm{X}}> \\
\mathrm{nT}\end{array}$ & $\begin{array}{r}<B_{\mathrm{y}}> \\
\mathrm{nT}\end{array}$ & $\begin{array}{r}<B_{\mathrm{Z}}> \\
\mathrm{nT}\end{array}$ \\
\hline $\mathrm{A}$ & $\begin{array}{c}43 \\
21: 48\end{array}$ & $\begin{array}{c}43 \\
22: 09\end{array}$ & $\begin{array}{c}43 \\
20: 39\end{array}$ & $\begin{array}{c}43 \\
20: 59\end{array}$ & $\begin{array}{c}43 \\
14: 08\end{array}$ & $\begin{array}{c}43 \\
15: 08\end{array}$ & 0.196 & -0.845 & -0.238 \\
\hline B & $\begin{array}{c}44 \\
00: 48\end{array}$ & $\begin{array}{c}44 \\
01: 08\end{array}$ & $\begin{array}{c}43 \\
23: 39\end{array}$ & $\begin{array}{c}43 \\
23: 59\end{array}$ & $\begin{array}{c}43 \\
17: 08\end{array}$ & $\begin{array}{c}43 \\
18: 08\end{array}$ & 0.338 & -0.831 & -0.106 \\
\hline $\mathrm{C}$ & $\begin{array}{c}45 \\
07: 10\end{array}$ & $\begin{array}{c}45 \\
07: 30\end{array}$ & $\begin{array}{c}45 \\
06: 01\end{array}$ & $\begin{array}{c}45 \\
06: 21\end{array}$ & $\begin{array}{c}44 \\
23: 31\end{array}$ & $\begin{array}{c}45 \\
00: 31\end{array}$ & -0.106 & 0.282 & 0.252 \\
\hline $\mathrm{D}$ & $\begin{array}{c}46 \\
05: 33\end{array}$ & $\begin{array}{c}46 \\
05: 53\end{array}$ & $\begin{array}{c}46 \\
04: 24\end{array}$ & $\begin{array}{c}46 \\
04: 44\end{array}$ & $\begin{array}{c}45 \\
21: 53\end{array}$ & $\begin{array}{c}45 \\
22: 53\end{array}$ & -0.062 & 0.121 & 0.152 \\
\hline
\end{tabular}

these intervals for each image are given in Table 1 (columns 2 and 3), together with the start and end times of the light emission at Saturn (columns 4 and 5). The intervals used to obtain the $1 \mathrm{~h}$-averaged IMF vectors are also given (columns 6 and 7), lagged by $\sim 6 \mathrm{~h}$ for all images as discussed above, corresponding to the blue striped intervals marked with the corresponding image label in Fig. 2. The last three columns of the table (8 to 10) then give the averaged KSM IMF components. It is notable in particular that IMF $B_{\mathrm{Z}}$ is negative for images $\mathrm{A}$ and $\mathrm{B}$, and positive for $\mathrm{C}$ and $\mathrm{D}$.

In Fig. 4 we present the projection of magnetospheric magnetic field lines in the $\mathrm{X}-\mathrm{Z}$ plane originating from the planet in the noon-midnight meridian for the above model parameters and KSM IMF components marked at the top of each plot. In the left-hand columns the IMF penetration coefficients $k_{\mathrm{S}}=0.8$, while in the right-hand columns $k_{\mathrm{s}}=0.2$. We note that the field lines on the nightside of the planet terminate where they intersect the paraboloid magnetopause. It can be seen that for southward and northward IMF the magnetospheric magnetic field structures are quite different, and that this fact is true even for rather modest IMF magnitudes less than $1 \mathrm{nT}$. It can also be seen that decreasing values of $k_{\mathrm{S}}$ lead to growth of the volume of the magnetosphere occupied by open and closed field lines. Overall, Fig. 4 shows that rather small variations in the IMF components within the model (at the level of a tenth of nT) lead to modifications of the large-scale magnetospheric magnetic field. As a consequence, corresponding changes in the shape and size of the open field line region in the ionosphere should also be expected, as we now go on to show.

\section{Comparison with HST auroral observations}

We now compare the calculated open field line region at the ionospheric level with the corresponding HST auroral images, as shown in Fig. 5. Each image consists of the sum of nine consecutive $100 \mathrm{~s}$ images of Saturn's southern oval obtained using the F115LP longpass filter over the $\sim 20 \mathrm{~min}$ intervals specified in Table 1, that have been combined together to increase signal-to-noise. The image identifier and start time are given at the top of each panel. The images are projected onto a spheroidal surface $1100 \mathrm{~km}$ above the 1-bar level (the altitude of peak brightness of the auroras, see Gérard et al., 2009), with noon at the bottom and dusk to the right. For further information about the reduction of these images see Clarke et al. (2009). Because the subEarth latitude on Saturn was $-8^{\circ}$, only the dayside portion of the southern oval is well-observed (and none of the northern oval), though even in that case the poleward boundary of the oval is somewhat obscured due to the finite height of the auroral curtain. As indicated above, images A and B obtained on DOY 43 and 44 shortly after the interplanetary shock is thought to have arrived at Saturn (Clarke et al., 2009) show bright auroras on the dawnside, reaching to high latitudes in this sector. By contrast, in the images obtained on DOY 45 and 46 the oval is less bright and the poleward boundary has moved equatorward in the dawn sector, such that the oval overall appears thinner and more circular.

The solid lines superimposed on each image are the calculated open field regions, where the red line is for IMF penetration coefficient $k_{\mathrm{s}}=0.8$ and the yellow for $k_{\mathrm{s}}=0.2$. The approximate corresponding IMF vectors are given at the bottom of each image (see Table 1). Considering first the results for $k_{\mathrm{s}}=0.8$, and comparing with Fig. 3, it can be seen for case $A$ that while the size of the ionospheric open field line region is expected to be reduced by the southward IMF conditions prevailing $\left(B_{\mathrm{Z}} \approx-0.2 \mathrm{nT}\right)$, it is also increased by the strong IMF Y-component $\left(B_{\mathrm{y}} \approx-0.8 \mathrm{nT}\right)$, such that overall the open region remains quite large. For case $B$, IMF $B_{\mathrm{y}}$ does not change significantly, but the averaged southward component becomes less $\left(B_{\mathrm{Z}} \approx-0.1 \mathrm{nT}\right)$, leading to a small increase in the area of the open field region. This increase 
a) $B_{\mathrm{x}}=-0.5 \mathrm{nT} ; B_{\mathrm{y}}=0.001 \mathrm{nT} ; B_{\mathrm{z}}=0.8 \mathrm{nT}$

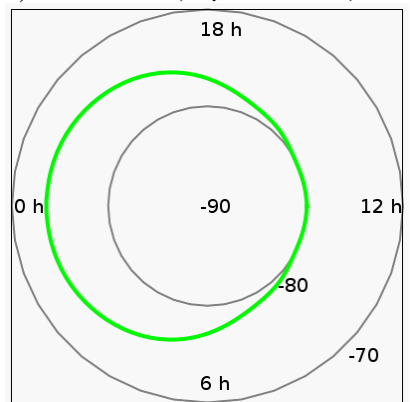

c) $B_{\mathrm{x}}=-0.5 \mathrm{nT} ; B_{\mathrm{y}}=0.001 \mathrm{nT} ; B_{\mathrm{z}}=-0.8 \mathrm{nT}$

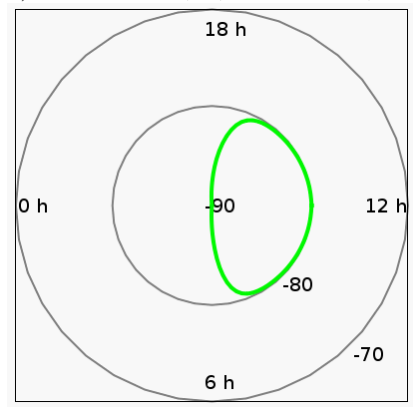

e) $B_{\mathrm{x}}=0.001 \mathrm{nT} ; B_{\mathrm{y}}=-0.5 \mathrm{nT} ; B_{\mathrm{z}}=0.8 \mathrm{nT}$

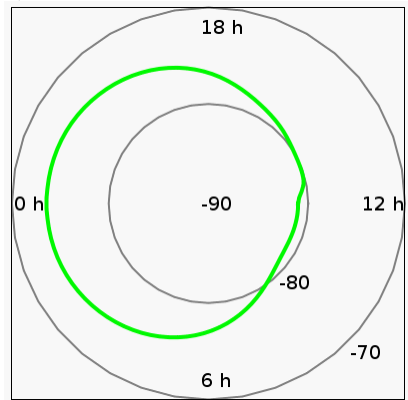

g) $B_{\mathrm{x}}=0.001 \mathrm{nT} ; B_{\mathrm{y}}=-0.5 \mathrm{nT} ; B_{\mathrm{z}}=-0.8 \mathrm{nT}$

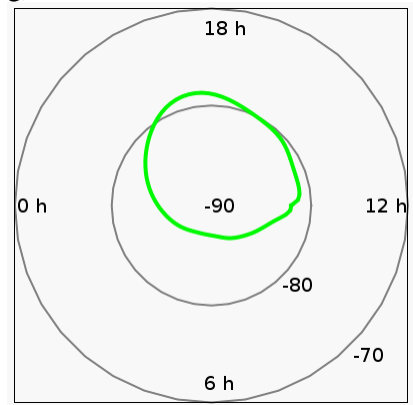

b) $B_{\mathrm{x}}=0.5 \mathrm{nT} ; B_{\mathrm{y}}=0.001 \mathrm{nT} ; B_{\mathrm{z}}=0.8 \mathrm{nT}$

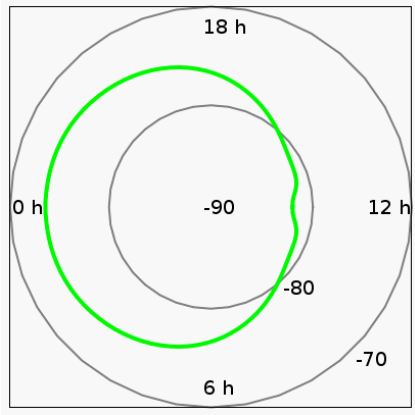

d) $B_{\mathrm{x}}=0.5 \mathrm{nT} ; B_{\mathrm{y}}=0.001 \mathrm{nT} ; B_{\mathrm{z}}=-0.8 \mathrm{nT}$

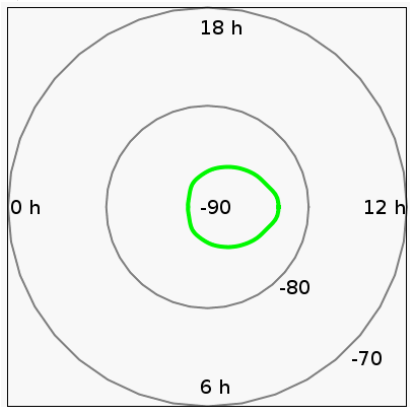

f) $B_{\mathrm{x}}=0.001 \mathrm{nT} ; B_{\mathrm{y}}=0.5 \mathrm{nT} ; B_{\mathrm{z}}=0.8 \mathrm{nT}$

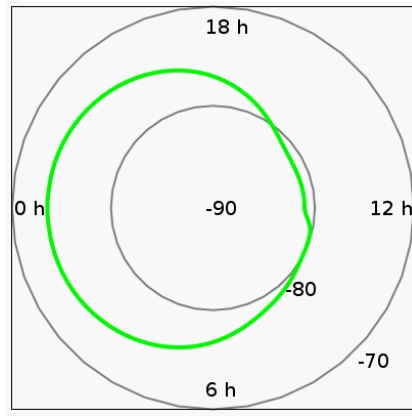

h) $B_{\mathrm{x}}=0.001 \mathrm{nT} ; B_{\mathrm{y}}=0.5 \mathrm{nT} ; B_{\mathrm{z}}=-0.8 \mathrm{nT}$

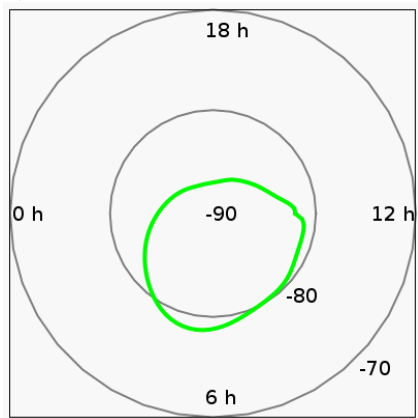

Fig. 3. Plots showing the effect of each KSM IMF component on the ionospheric open field line region in the Southern Hemisphere, where noon is on the right of each plot, and dusk at the top. Latitude circles are shown with $10^{\circ}$ steps, while green curves show the calculated open-closed field line boundary. The corresponding IMF vector is given at the top of each plot, and we have taken the penetration coefficient $k_{\mathrm{S}}=0.8$. Plots (a) and (b) show the influence of $B_{\mathrm{X}}$ for $B_{\mathrm{Z}}>0$, (c) and (d) the influence of $B_{\mathrm{X}}$ for $B_{\mathrm{Z}}<0$, (e) and (f) the influence of $B_{\mathrm{y}}$ for $B_{\mathrm{Z}}>0$, and (g) and (h) the influence of $B_{\mathrm{y}}$ for $B_{\mathrm{Z}}<0$.

continues for case $C$ due to a transition to relatively strong northward IMF conditions $\left(B_{\mathrm{z}} \approx 0.3 \mathrm{nT}\right)$, despite the smaller value of $B_{\mathrm{y}} \approx 0.3 \mathrm{nT}$. For image $D$ the open flux is slightly reduced again due to the smaller value of $B_{\mathrm{Z}}$. For $k_{\mathrm{S}}=0.2$ the open field area is reduced throughout compared with $k_{\mathrm{S}}=0.8$, while the effect of IMF $B_{\mathrm{y}}$ is more pronounced, the open area 
Case $A ; k \mathrm{~s}=0.8 ; B_{\mathrm{x}} \approx 0.2, B_{\mathrm{y}} \approx-0.8, B_{\mathrm{z}} \approx-0.2 \mathrm{nT}$

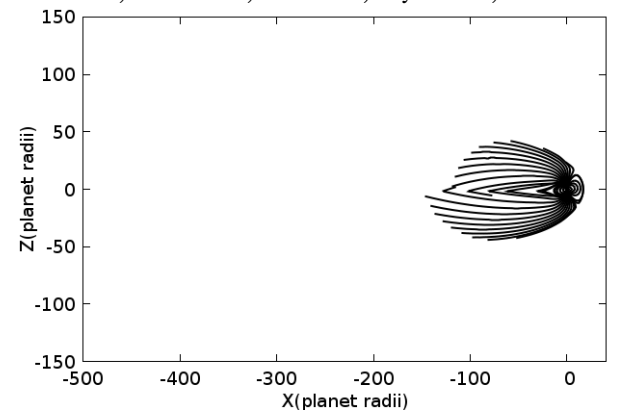

Case $B ; k \mathrm{~s}=0.8 ; B_{\mathrm{x}} \approx 0.3, B_{\mathrm{y}} \approx-0.8, B_{\mathrm{z}} \approx-0.1 \mathrm{nT}$

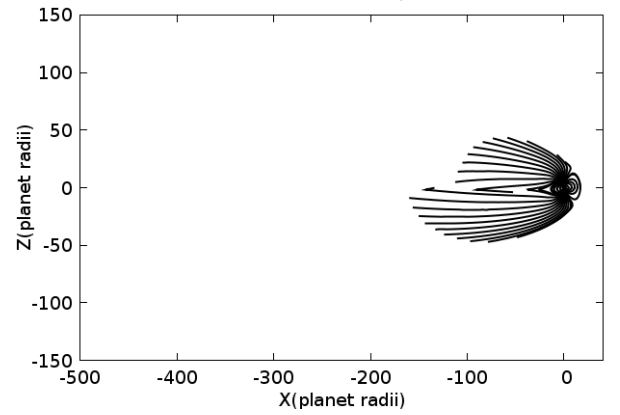

Case $C ; k \mathrm{~s}=0.8 ; B_{\mathrm{x}} \approx-0.1, B_{\mathrm{y}} \approx 0.3, B_{\mathrm{z}} \approx 0.3 \mathrm{nT}$

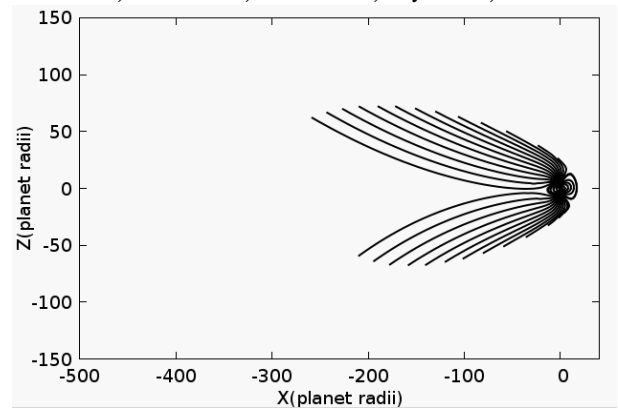

Case $D ; k \mathrm{~s}=0.8 ; B_{\mathrm{x}} \approx 0.1, B_{\mathrm{y}} \approx 0.1, B_{\mathrm{z}} \approx 0.2 \mathrm{nT}$

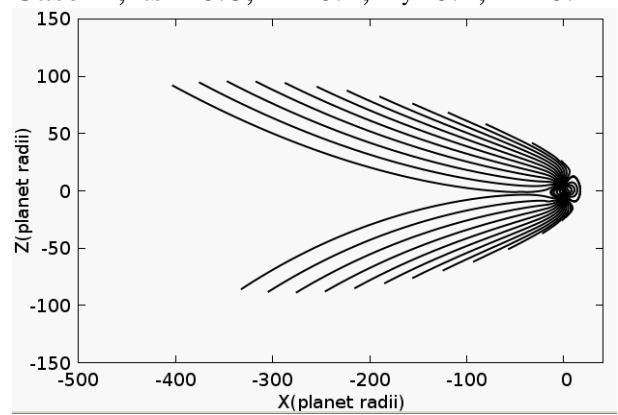

Case $A ; k \mathrm{~s}=0.2 ; B_{\mathrm{x}} \approx 0.2, B_{\mathrm{y}} \approx-0.8, B_{\mathrm{z}} \approx-0.2 \mathrm{nT}$

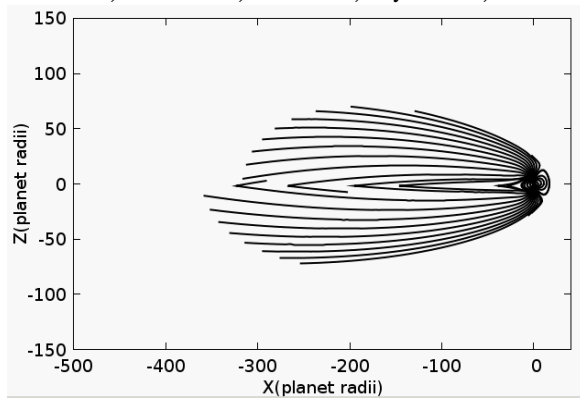

Case $B ; k \mathrm{~s}=0.2 ; B_{\mathrm{x}} \approx 0.3, B_{\mathrm{y}} \approx-0.8, B_{\mathrm{z}} \approx-0.1 \mathrm{nT}$

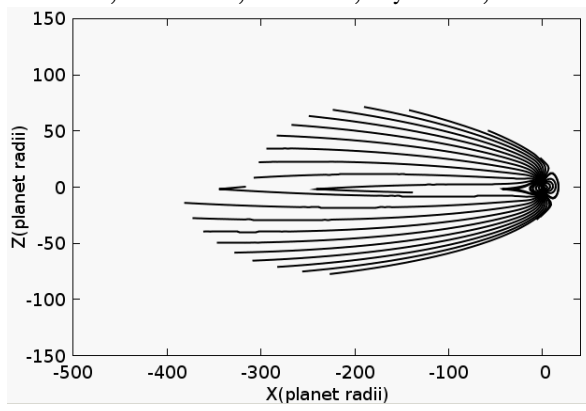

Case $C ; k \mathrm{~s}=0.2 ; B_{\mathrm{x}} \approx-0.1, B_{\mathrm{y}} \approx 0.3, B_{\mathrm{z}} \approx 0.3 \mathrm{nT}$

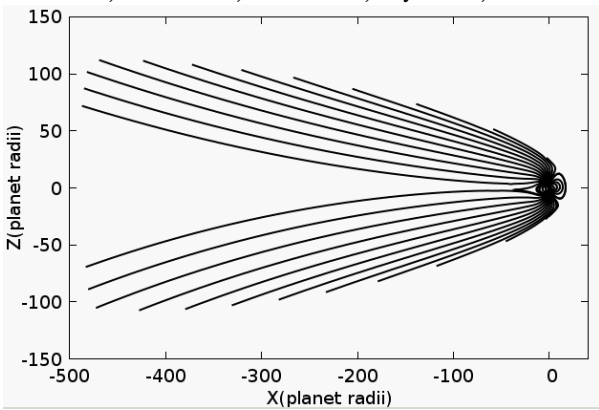

Case $D ; k \mathrm{~s}=0.2 ; B_{\mathrm{x}} \approx 0.1, B_{\mathrm{y}} \approx 0.1, B_{\mathrm{z}} \approx 0.2 \mathrm{nT}$

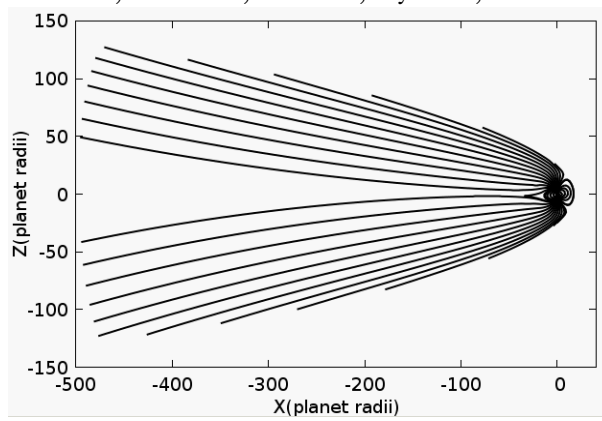

Fig. 4. Plots showing field lines emerging from Saturn's ionosphere in the noon-midnight meridian for IMF vectors corresponding to HST images $\mathrm{A}, \mathrm{B}, \mathrm{C}$, and $\mathrm{D}$, as given at the top of each plot. In the left-hand plots $k_{\mathrm{S}}=0.8$, while on the right $k_{\mathrm{S}}=0.2$. Field lines on the nightside terminate where they intersect the magnetopause.

being shifted significantly towards dusk in cases A and B for negative $B_{\mathrm{y}}$, and towards dawn in cases $\mathrm{C}$ and $\mathrm{D}$ for positive $B_{\mathrm{y}}$. In case $\mathrm{D}$ we see that the open area decreases significantly compared with the other cases due to the small values of both IMF $B_{\mathrm{y}}$ and $B_{\mathrm{z}}$. These results thus indicate that even variations in the direction of an IMF of modest magnitude can significantly influence the location of the open field line region in Saturn's polar ionosphere.

Now comparing the model boundaries with the auroral emissions in the images, it can be seen in each case that 
A $04321: 47: 38$ (UT at HST)

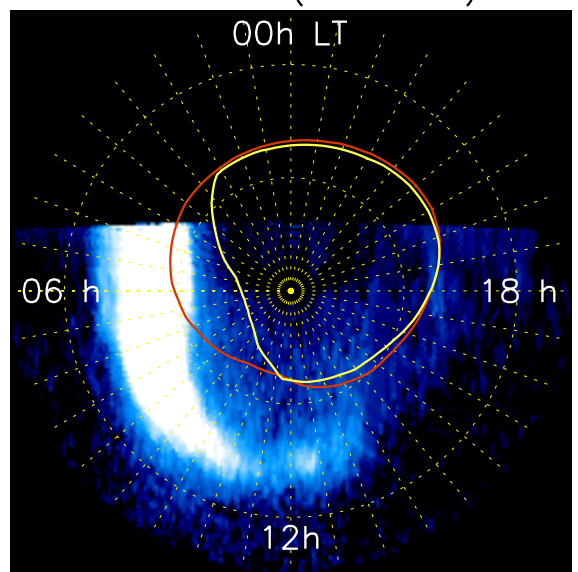

Red: $k_{s}=0.8$, Yellow: $k_{s}=0.2$ $\mathrm{B}(\mathrm{x}, \mathrm{y}, \mathrm{z})=(0.2,-0.8,-0.2) \mathrm{nT}$

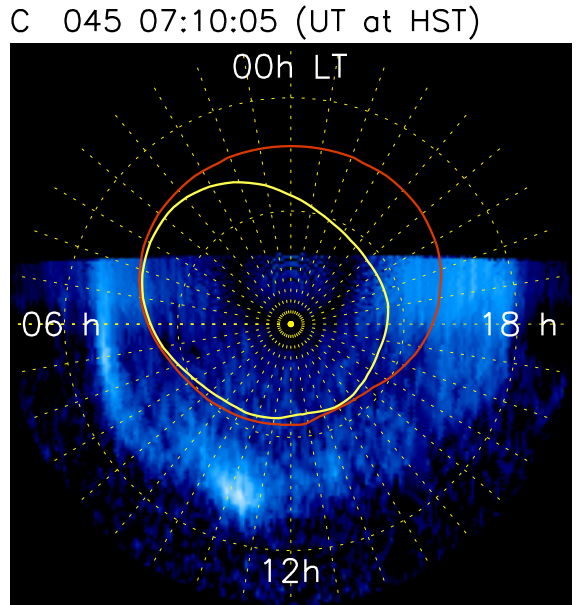

Red: $k_{s}=0.8$, Yellow: $k_{s}=0.2$

$$
B(x, y, z)=(-0.1,0.3,0.3) n T
$$

B $04400: 47: 44$ (UT at HST)

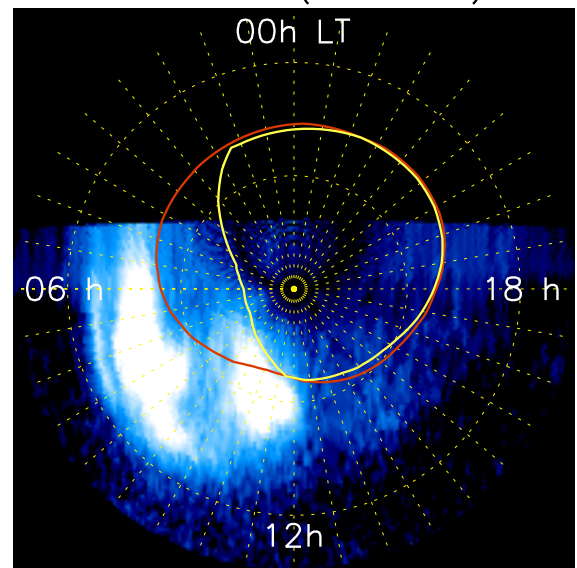

Red: $k_{s}=0.8$, Yellow: $k_{s}=0.2$

$B(x, y, z)=(0.3,-0.8,-0.1) n T$

D $04605: 32: 52$ (UT at HST)

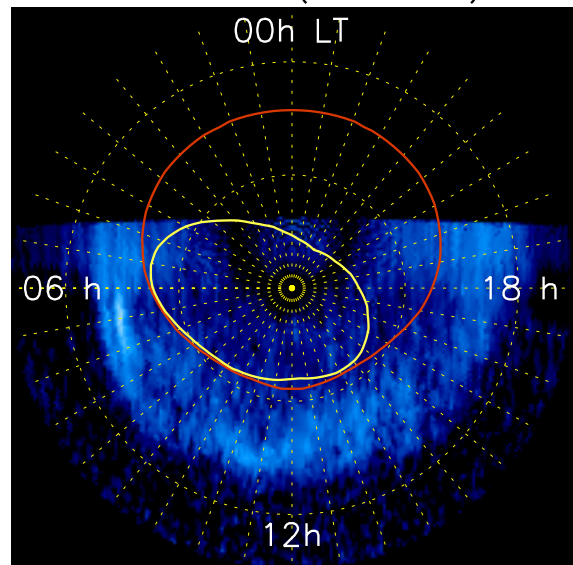

Red: $k_{s}=0.8$, Yellow: $k_{s}=0.2$

$B(x, y, z)=(-0.1,0.1,0.2) n T$

Intensity (kR)

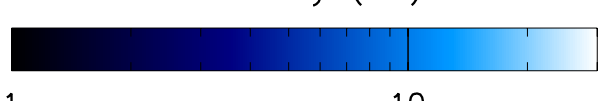

10

Fig. 5. Plots showing HST UV images of Saturn's southern auroras, projected onto a spheroidal surface $1100 \mathrm{~km}$ above the 1 bar level, with noon at the bottom and dawn to the left. Image identifiers are given at the top of each panel together with the start time of the $\sim 20$ min combined exposure time as given in Table 1 . The superposed solid lines show the calculated open field regions for $k_{\mathrm{S}}=0.8$ (red) and $k_{\mathrm{S}}=0.2$ (yellow), using the corresponding averaged IMF vector determined from Cassini data, as indicated at the bottom of each panel.

the modelled open field region lies approximately within the poleward area bounded by the emission, with the model boundary lying adjacent to the poleward limit of the emissions. The open field area is somewhat smaller in the upper panels for southward IMF and larger in the lower panels for northward IMF, at least for $k_{\mathrm{s}}=0.8$, despite the equatorward boundary being relatively stable. For $k_{\mathrm{S}}=0.2$ the value of IMF $B_{\mathrm{y}}$ is also strongly influential. Overall, the results support the view that Saturn's auroras are associated with the boundary of open field lines as proposed previously by Cowley and Bunce (2003), Cowley et al. (2004a, b), and Bunce et al. (2008), the auroras lying adjacent to and equatorward of the model boundary. 


\section{Conclusions}

In this paper we have further studied the relationship between the dynamics of Saturn's auroral emissions and variations in the IMF in the upstream solar wind, following discussions of the January 2004 joint Cassini-HST imaging campaign data by Belenkaya et al. $(2007,2008)$. While the latter campaign provided an important initial data set for study, the large $\sim 1300 R_{\mathrm{S}}$ displacement between the spacecraft and the planet with a related $\sim 17 \mathrm{~h}$ radial propagation delay results in considerable uncertainties in the identification of detailed correspondences between the interplanetary data and the auroral images. Here, however, we have investigated a subsequent data set, unique to date, in which auroral images were obtained when Cassini was located immediately upstream of Saturn's bow shock, in which the related timing uncertainties were consequently much reduced.

Specifically, we have investigated images obtained during the February 2008 HST campaign (DOY 43-46), that can be related to IMF data obtained by the Cassini spacecraft near apoapsis on Revs 58 and 59, just upstream of Saturn's dayside bow shock. From continuous propagated near-Earth solar wind data presented by Clarke et al. (2009), the interval is believed to have been associated with a compression of the magnetosphere by an interplanetary shock on DOY 43, following which the magnetosphere was further compressed on DOYs 44 and 45 , with solar wind dynamic pressures peaking at $\sim 0.1 \mathrm{nPa}$ on these days. Here we have combined suitably lagged Cassini IMF data with the paraboloid model of Saturn's magnetosphere, to calculate the open field region in Saturn's southern ionosphere as imaged by the HST. The magnetic model has fixed parameters appropriate to relatively compressed conditions $(\sim 0.08 \mathrm{nPa})$ apart from the penetrating IMF vector, so that the effects of variations in the latter can be studied in isolation. However, we have also repeated the calculations for a magnetic model appropriate to intermediate conditions $(\sim 0.03 \mathrm{nPa})$, and have verified that the results are not sensitively dependent on this choice.

With regard to the lag time employed for the Cassini IMF data relative to the HST images, three propagation and response time effects were considered. These are the one-way light travel time between Saturn and the HST $(\sim 1 \mathrm{~h})$, the solar wind propagation time between Cassini and the reconnection regions on Saturn's magnetopause $(\sim 0.5 \mathrm{~h})$, and the auroral and flow response time in Saturn's ionosphere resulting from changes in the IMF $(\sim 5.5 \mathrm{~h})$. The latter response time was estimated by suitably scaling the related flow response time observed in the Earth's system. The lagged IMF data were then averaged over $1 \mathrm{~h}$ intervals in order to obtain a reasonable representative value of the IMF relating to each auroral image. These vectors were then employed within the model magnetosphere to determine the location of the open field region in the southern ionosphere corresponding to the HST images. It was found that although the magnitude of the IMF was rather small during the interval, $\sim 0.2-0.9 \mathrm{nT}$, compared with $\sim 2 \mathrm{nT}$ during the January 2004 campaign, a significant open field region was still present in the model field, which responded in size and position with the orientation of the IMF vector.

Comparison of the auroral images with the modelled open field regions then shows that they bear a consistent relationship with each other. The open field regions are generally contained inside the auroral oval, with the IMF-modulated open-closed field line boundary lying adjacent to the poleward boundary of the aurora. This result is important for the physics of Saturn's auroras, the origins of which are still a matter of considerable debate. It is also clearly in agreement with the conclusions reached previously by Belenkaya et al. $(2007,2008)$ from analysis of the January 2004 campaign observations. However, the unique data set examined here in which Cassini was located just upstream of Saturn's bow shock during the imaging intervals now place these conclusions on a much firmer basis than those derived earlier.

Acknowledgements. Work at the Institute of Nuclear Physics, Moscow State University was supported by Royal Society (London)/RFBR Grant No 09-02-92603-KO_a, and by RFBR Grants 07-05-00529 and 09-05-00798. Work at Leicester was also supported by the Royal Society/RFBR Grant (JP080836), and by STFC grants PP/E000983/1 and ST/H002480/1. This work employs observations made with the NASA/ESA Hubble Space Telescope, obtained at the Space Telescope Science Institute, which is operated by AURA, Inc. for NASA.

Topical Editor R. Nakamura thanks C. Arridge and another anonymous referee for their help in evaluating this paper.

\section{References}

Alexeev, I. I., Kalegaev, V. V., Belenkaya, E. S., Bobrovnikov, S. Y., Bunce, E. J., Cowley, S. W. H., and Nichols, J. D.: A global magnetic model of Saturn's magnetosphere, and a comparison with Cassini SOI data, Geophys. Res. Lett., 33, L08101, doi:10.1029/2006GL025896, 2006.

Badman, S. V. and Cowley, S. W. H.: Significance of Dungey-cycle flows in Jupiter's and Saturn's magnetospheres, and their identification on closed equatorial field lines, Ann. Geophys., 25, 941951, doi:10.5194/angeo-25-941-2007, 2007.

Badman, S. V., Bunce, E. J., Clarke, J. T., Cowley, S. W. H., Gérard, J.-C., Grodent, D., and Milan, S. E.: Open flux estimates in Saturn's magnetosphere during the January 2004 Cassini-HST campaign, and implications for reconnection rates: J. Geophys. Res., 110, A11216, doi:10.1029/2005JA011240, 2005.

Badman, S. V., Cowley, S. W. H., Gérard, J.-C., and Grodent, D.: A statistical analysis of the location and width of Saturn's southern auroras, Ann. Geophys., 24, 3533-3545, doi:10.5194/angeo-243533-2006, 2006.

Badman, S. V., Cowley, S. W. H., Lamy, L., Cecconi, B., and Zarka, P.: Relationship between solar wind corotating interaction regions and the phasing and intensity of Saturn kilometric radiation bursts, Ann. Geophys., 26, 3641-3651, doi:10.5194/angeo26-3641-2008, 2008. 
Belenkaya, E. S., Cowley, S. W. H., and Alexeev, I. I.: Saturn's aurora in the January 2004 events, Ann. Geophys., 24, 16491663, doi:10.5194/angeo-24-1649-2006, 2006a.

Belenkaya, E. S., Alexeev, I. I., Kalegaev, V. V., and Blokhina, M. S.: Definition of Saturn's magnetospheric model parameters for the Pioneer 11 flyby, Ann. Geophys., 24, 1145-1156, doi:10.5194/angeo-24-1145-2006, 2006b.

Belenkaya, E. S., Alexeev, I. I., Blokhina, M. S., Cowley, S. W. H., Badman, S. V., Kalegaev, V. V., and Grigoryan, M. S.: IMF dependence of the open-closed field line boundary in Saturn's ionosphere, and its relation to the UV auroral oval observed by the Hubble Space Telescope, Ann. Geophys., 25, 1215-1226, doi:10.5194/angeo-25-1215-2007, 2007.

Belenkaya, E. S., Cowley, S. W. H., Badman, S. V., Blokhina, M. S., and Kalegaev, V. V.: Dependence of the open-closed field line boundary in Saturn's ionosphere on both the IMF and solar wind dynamic pressure: comparison with the UV auroral oval observed by the HST, Ann. Geophys., 26, 159-166, doi:10.5194/angeo-26-159-2008, 2008.

Bunce, E. J., Cowley, S. W. H., Jackman, C. M., Clarke, J. T., Crary, F. J., and Dougherty, M. K.: Cassini observations of the interplanetary medium upstream of Saturn and their relation to Hubble Space Telescope aurora data, Adv. Space Res., 38, 806-814, 2006.

Bunce, E. J., Arridge, C. S., Clarke, J. T., Coates, A. J. S., Cowley, W. H., Dougherty, M. K., Gérard, J.-C., Grodent, D., Hansen, K. C., Nichols, J. D., Southwood, D. J., and Talboys, D. L.: Origin of Saturn's aurora: Simultaneous observations by Cassini and the Hubble Space Telescope, J. Geophys. Res., 113, A09209, doi:10.1029/2008JA013257, 2008.

Clarke, J. T., Gérard, J.-G., Grodent, D., Wannawichian, S., Gustin, J., Connerney, J., Crary, F., Dougherty, M., Kurth, W., Cowley, S. W. H., Bunce, E. J., Hill, T., and Kim, J.: Morphological differences between Saturn's ultraviolet aurorae and those of Earth and Jupiter, Nature, 433, 717-719, doi:10.1038/nature03331, 2005.

Clarke, J. T., Nichols, J., Gérard, J.-C., Grodent, D., Hansen, K. C., Kurth, W., Gladstone, G. R., Duval, J., Wannawichian, S., Bunce, E. J., Cowley, S. W. H., Crary, F., Dougherty, M., Lamy, L., Mitchell, D., Pryor, W., Retherford, K., Stallard, T., Zieger, B., Zarka, P., and Cecconi, B.: Response of Jupiter's and Saturn's auroral activity to the solar wind, J. Geophys. Res., 114, A05210, doi:10.1029/2008JA013694, 2009.

Clauer, C. R. and Friis-Christensen, E.: High-latitude dayside electric fields and currents during strong northward interplanetary magnetic field: Observations and model simulation, J. Geophys. Res., 93, 2749-2757, 1988.

Cowley, S. W. H. and Bunce, E. J.: Origin of the main auroral oval in Jupiter's coupled magnetosphere-ionosphere system, Planet. Space Sci., 49, 1067-1088, 2001.

Cowley, S. W. H. and Bunce, E. J.: Corotation-driven magnetosphere-ionosphere coupling currents in Saturns magnetosphere and their relation to the auroras, Ann. Geophys., 21, 1691-1707, doi:10.5194/angeo-21-1691-2003, 2003.

Cowley, S. W. H., Bunce, E. J., and Prangé, R.: Saturn's polar ionospheric flows and their relation to the main auroral oval, Ann. Geophys., 22, 1379-1394, doi:10.5194/angeo-22-13792004, 2004a.

Cowley, S. W. H., Bunce, E. J., and O'Rourke, J. M.: A simple quantitative model of plasma flows and currents in
Saturn's polar ionosphere, J. Geophys. Res., 109, A05212, doi:10.1029/2003JA010375, 2004b.

Cowley, S. W. H., Badman, S. V., Bunce, E. J., Clarke, J. T., Gérard, J.-C., Grodent, D., Jackman, C. M., Milan, S. E., and Yeoman, T. K.: Reconnection in a rotation-dominated magnetosphere and its relation to Saturn's auroral dynamics, J. Geophys. Res., 110, A02201, doi:10.1029/2004JA010796, 2005.

Cowley, S. W. H., Arridge, C. S., Bunce, E. J., Clarke, J. T., Coates, A. J., Dougherty, M. K., Gérard, J.-C., Grodent, D., Nichols, J. D., and Talboys, D. L.: Auroral current systems in Saturn's magnetosphere: comparison of theoretical models with Cassini and HST observations, Ann. Geophys., 26, 2613-2630, doi:10.5194/angeo-26-2613-2008, 2008.

Crary, F. J., Clark, J. T., Dougherty, M. K., Hanlon, P. G., Hansen, K. C., Steinberg, J. T., Barrachlough, B. L., Coates, A. J., Gérard, J.-C., Grodent, D., Kurth, W. S., Mitchell, D. G., Rymer, A. M., and Young, D. T.: Solar wind dynamic pressure and electric field as the main factors controlling Saturn's auroras, Nature, 433, 720-722, doi:10.1038/nature03333, 2005.

Dougherty, M. K., Kellock, S., Southwood, D. J., Balogh, A., Smith, E. J., Tsurutani, B. T., Gerlach, B., Glassmeier, K.-H., Gleim, F., Russell, C. T., Erdos, G., Neubauer, F. M., and Cowley, S. W. H.: The Cassini magnetic field investigation, Space Sci. Rev., 114, 331-383, doi:10.1007/s11214-004-1432-2, 2004.

Gérard, J.-C., Grodent, D., Gustin, J., and Saglam, A.: Characteristics of Saturn's FUV aurora observed with the Space Telescope Imaging Spectrograph, J. Geophys. Res., 109, A09207, doi:10.1029/2004JA010513, 2004.

Gérard, J.-C., Bondford, B., Gustin, J., Grodent, D., Clarke, J. T., Bisikalo, D., and Shematovich, V.: Altitude of Saturn's aurora and its implications for the characteristic energy of precipitated electrons, Geophys. Res. Lett., 36, L02202, doi:10.1029/2008GL036554, 2009.

Grodent, D., Gérard, J.-C., Cowley, S. W. H., Bunce, E. J., and Clarke, J. T.: Variable morphology of Saturn's southern ultraviolet aurora, J. Geophys. Res., 110, A07215, doi:10.1029/2004JA010983, 2005.

Hairston, M. R. and Heelis, R. A.: Response time of the polar ionospheric convection pattern to changes in the north-south direction of the IMF, Geophys. Res. Lett., 22, 631-634, 1995.

Huang, C.-S., Murr, D., Sofko, G. J., Hughes, W. J., and Moretto, T.: Ionospheric convection response to changes of interplanetary magnetic field component during strong By component, J. Geophys. Res., 105, 5231-5243, 2000.

Jackman, C. M., Achilleos, N., Bunce, E. J., Cecconi, B., Clarke, J. T., Cowley, S. W. H., Kurth, W. S., and Zarka, P.: Interplanetary conditions and magnetospheric dynamics during the Cassini orbit insertion fly-through of Saturn's magnetosphere, J. Geophys. Res., 110, A10212, doi:10.1029/2005JA011054, 2005.

Kanani, S. J., Arridge, C. S., Jones, G. H., Fazakerley, A. N., McAndrews, H. J., Sergis, N., Krimigis, S. M., Dougherty, M. K., Coates, A. J., Young, D. T., Hansen, K. C., and Krupp, N.: A new form of Saturn's magnetopause using a dynamic pressure model, based on in-situ, multi-instrument Cassini measurements, J. Geophys. Res., 115, A06207, doi:10.1029/2009JA014262, 2010.

Kurth, W. S., Gurnett, D. A., Clarke, J. T., Zarka, P., Desch, M. D., Kaiser, M. L., Cecconi, B., Lecacheux, A., Farrell, W. M., Galopeau, P., Gérard, J.-C., Grodent, D., Prangé, R., Dougherty, M. K., and Crary, F. J.: An Earth-like corre- 
spondence between Saturn's ultraviolet auroral features and radio emission, Nature, 433, 722-725, doi:10.1038/nature03334, 2005.

Masters, A., Achilleos, N., Dougherty, M. K., Slavin J. A., Hospodarsky, G. B., Arridge, C. S., and Coates, A. J.: An empirical model of Saturn's bow shock: Cassini observations of shock location and shape, J. Geophys. Res., 113, A10210, doi:10.1029/2008JA013276, 2008.

Milan, S. E., Boakes, P. D., and Hubert, B.: Response of the expanding/contracting polar cap to weak and strong solar wind driving: Implications for substorm onset, J. Geophys. Res., 113, A09215, doi:10.1029/2008JA013340, 2008.

Nichols, J. D. and Cowley, S. W. H.: Magnetosphereionosphere coupling currents in Jupiter's middle magnetosphere: effect of precipitation-induced enhancement of the ionospheric Pedersen conductivity, Ann. Geophys., 22, 1799-1827, doi:10.5194/angeo-22-1799-2004, 2004.

Ridley, A. J., Lu, G., Clauer, C. R., and Papitashvili, V. O.: Ionospheric convection during nonsteady interplanetary magnetic field conditions, J. Geophys. Res., 102, 14563-14579, 1997.
Ridley, A. J., Lu, G., Clauer, C. R., and Papitashvili, V. O.: A statistical study of the ionospheric convection response to changing interplanetary magnetic field conditions using the assimilative mapping of ionospheric electrodynamics technique, J., Geophys. Res., 103, 4023-4039, 1998a.

Rishbeth, H., Smith, P. R., Cowley, S. W. H., Willis, D. M., van Eyken, A. P., Bromage, B. J. I., and Crothers, S. R.: Ionospheric response to changes in the interplanetary magnetic field observed by EISCAT and AMPTE-UKS, Nature, 318, 451-452; doi:10.1038/318451a0, 1985.

Taylor, J. R., Yeoman, T. K., Lester, M., Buonsanto, M. J., Scali, J. L., Ruohoniemi, J. M., and Kelly, J. D.: Ionospheric convection during the magnetic storm of 20-21 March 1990, Ann. Geophys., 12, 1174-1191, doi:10.1007/s00585-994-1174-1, 1994.

Todd, H., Cowley, S. W. H., Lockwood, M., Willis, D. M., and Luehr, H.: Response time of the high-latitude dayside ionosphere to sudden changes in the north-south component of the IMF, Planet. Space Sci., 36, 1415-1428, 1988. 